\title{
COMPACT KÄHLER MANIFOLDS WITH AUTOMORPHISM GROUPS OF MAXIMAL RANK
}

\author{
DE-QI ZHANG
}

\begin{abstract}
For an automorphism group $G$ on an $n$-dimensional $(n \geq 3)$ normal projective variety or a compact Kähler manifold $X$ so that $G$ modulo its subgroup $N(G)$ of null entropy elements is an abelian group of maximal rank $n-1$, we show that $N(G)$ is virtually contained in $\operatorname{Aut}_{0}(X)$, the $X$ is a quotient of a complex torus $T$ and $G$ is mostly descended from the symmetries on the torus $T$, provided that both $X$ and the pair $(X, G)$ are minimal.
\end{abstract}

\section{INTRODUCTION}

We work over the field $\mathbb{C}$ of complex numbers. For a linear transformation $L$ on a finite-dimensional vector space $V$ over $\mathbb{C}$ or its subfields, its spectral radius is defined as

$$
\rho(L):=\max \{|\lambda| ; \lambda \in \mathbb{C} \text { is an eigenvalue of } L\} .
$$

Let $X$ be a compact complex Kähler manifold and $Y$ a normal projective variety, and let $g \in \operatorname{Aut}(X)$ and $f \in \operatorname{Aut}(Y)$. Define the (topological) entropy $h(*)$ and first dynamical degrees $d_{1}(*)$ as

$$
\begin{aligned}
h(g) & :=\log \rho\left(g^{*} \mid \bigoplus_{i \geq 0} H^{i}(X, \mathbb{C})\right), \\
d_{1}(g) & :=\rho\left(g^{*} \mid H^{2}(X, \mathbb{C})\right)\left(=\rho\left(g^{*} \mid H^{1,1}(X)\right)\right), \\
d_{1}(f): & =\rho\left(f^{*} \mid \operatorname{NS}_{\mathbb{C}}(Y)\right),
\end{aligned}
$$

where $\mathrm{NS}_{\mathbb{C}}(Y):=\mathrm{NS}(Y) \otimes_{\mathbb{Z}} \mathbb{C}$ is the complexified Neron-Severi group. By the fundamental work of Gromov and Yomdin, the above definition of entropy is equivalent to its original definition (cf. [6, §2.2] and the references therein). Further, when $Y$ is smooth, the above two definitions of $d_{1}(*)$ coincide; for $\mathbb{Q}$-factorial $Y$ (cf. [10, 0.4(1)]), we have $d_{1}(f)=d_{1}(\widetilde{f})$, where $\widetilde{f}$ is the lifting of $f$ to the one on an Aut $(Y)$-equivariant resolution of $Y$. We say that $\tau:=g$ or $f$ is of positive entropy (resp. null entropy) if $d_{1}(\tau)>1$ (resp. $d_{1}(\tau)=1$ ), or equivalently $h(\tau)>0$ (resp. $h(\tau)=0)$ in the case of a compact Kähler manifold.

We say that the induced action $G \mid H^{1,1}(X)$ is $Z$-connected if its Zariski-closure in $\mathrm{GL}\left(H^{1,1}(X)\right)$ is connected with respect to the Zariski topology; in this case, the null set

$$
N(G):=\{g \in G \mid g \text { is of null entropy }\}
$$

Received by the editors August 23, 2012.

2010 Mathematics Subject Classification. Primary 32H50, 37C85, 32M05, 14J50, 32Q15.

Key words and phrases. Automorphism group, Kähler manifold, Tits alternative, topological entropy.

The author was supported by an ARF of NUS. 
is a (necessarily normal) subgroup of $G$ (cf. [19, Theorem 1.2]). In [19, we have proved:

Theorem 1.1. Let $X$ be an $n$-dimensional $(n \geq 2)$ compact complex Kähler manifold and $G$ a subgroup of $\operatorname{Aut}(X)$. Then one of the following two assertions holds:

(1) $G$ contains a subgroup isomorphic to the non-abelian free group $\mathbb{Z} * \mathbb{Z}$, and hence $G$ contains subgroups isomorphic to non-abelian free groups of all countable ranks.

(2) There is a finite-index subgroup $G_{1}$ of $G$ such that the induced action $G_{1} \mid H^{1,1}(X)$ is solvable and Z-connected. Further, the subset

$$
N\left(G_{1}\right):=\left\{g \in G_{1} \mid g \text { is of null entropy }\right\}
$$

of $G_{1}$ is a normal subgroup of $G_{1}$ and the quotient group $G_{1} / N\left(G_{1}\right)$ is a free abelian group of rank $r \leq n-1$. We call this $r$ the rank of $G_{1}$ and denote it as $r=r\left(G_{1}\right)$.

Therefore, we are interested in the group $G \leq \operatorname{Aut}(X)$, where $G \mid H^{1,1}(X)$ is solvable and $Z$-connected and where the $\operatorname{rank} r(G)=\operatorname{dim} X-1$ (maximal value). In the following, denote by $\operatorname{Aut}_{0}(X)$ the identity connected component of $\operatorname{Aut}(X)$. A group virtually has a property $(\mathrm{P})$ if a finite-index subgroup of it has the property $(\mathrm{P})$.

A complex torus has lots of symmetries. Conversely, our main result, Theorem 1.2 (see also Theorem 2.1 for non-algebraic manifolds), says that the maximality $r(G)=\operatorname{dim} X-1$ occurs only when $X$ is a quotient of a complex torus $T$ and $G$ is mostly descended from the symmetries on the torus $T$.

The statement of Theorem 1.2 involves minimal varieties and canonical singularities, but our method uses only well-known and precisely referred facts in algebraic geometry rather than the technical part of the Minimal Model Program, and hence is accessible.

Below is our main result and the final one of our series of papers [19], [22] and [23].

Theorem 1.2. Let $X$ be an $n$-dimensional $(n \geq 3)$ normal projective variety and $G \leq \operatorname{Aut}(X)$ a subgroup such that the induced action $G \mid \mathrm{NS}_{\mathbb{C}}(X)$ is solvable and $Z$-connected and that the rank $r(G)=n-1$ (i.e., $G / N(G)=\mathbb{Z}^{\oplus n-1}$ ). Assume the following three conditions:

(i) X has at worst canonical, quotient singularitie@1 (cf. [10, Definition 2.34]).

(ii) $X$ is a minimal variety, i.e., the canonical divisor $K_{X}$ is nef (cf. [10, $0.4(3)])$.

(iii) The pair $(X, G)$ is minimal in the sense of Section 2.5 .

Then the following four assertions hold:

(1) The induced action $N(G) \mid \mathrm{NS}_{\mathbb{C}}(X)$ is a finite group.

(2) $G \mid \mathrm{NS}_{\mathbb{C}}(X)$ is a virtually free abelian group of rank $n-1$.

\footnotetext{
${ }^{1}$ In Theorem 1.2 the condition (i) can be weakened to: (i)' $X$ has at worst klt singularities and is smooth in codimention two. This is by the same proof of ours, taking the global index1 cover, and using Theorem 1.16 in the recent paper of D. Greb, S. Kebekus and T. Peternell: Étale fundamental groups of Kawamata log terminal spaces, flat sheaves, and quotients of Abelian varieties, arXiv:1307.5718.
} 
(3) Either $N(G)$ is a finite subgroup of $G$ and hence $G$ is a virtually free abelian group of rank $n-1$, or $X$ is an abelian variety and the group $N(G) \cap \operatorname{Aut}_{0}(X)$ has finite-index in $N(G)$ and is Zariski-dense in $\operatorname{Aut}_{0}(X)(\cong X)$.

(4) We have $X \cong T / F$ for a finite group $F$ acting freely outside a finite set of an abelian variety $T$. Further, for some finite-index subgroup $G_{1}$ of $G$, the action of $G_{1}$ on $X$ lifts to an action of $G_{1}$ on $T$.

For non-algebraic manifolds, a parallel result is given in Theorem 2.1

In 23], we assumed that (i) $G$ is abelian and (ii) the absence of pointwise $G$ fixed subvarieties of positive dimension or $G$-periodic rational curves or $Q$-tori. In the current paper, these two restrictions are replaced by the natural minimality condition on $X$ and the pair $(X, G)$, and that $G \mid \mathrm{NS}_{\mathbb{C}}(X)$ is solvable, the latter of which is natural in view of Theorem 1.1. The quotient singularities assumption in Theorem 1.2 is necessary because an effective characterization of the torus quotient is only available in dimension three by [16, where the bulk of the argument is to show that the variety has only quotient singularities.

The lack of the abelian-ness assumption on $G$ makes our argument much harder, for instance we cannot simultaneously diagonalize $G \mid \mathrm{NS}_{\mathbb{C}}(X)$ or find enough number of linearly independent common nef eigenvectors of $G$ as required in [6] for abelian groups.

Theorems [1.2 and 2.1 answer [19, Question 2.17], assuming the conditions here. When $G$ is abelian, the finiteness of $N(G)$ is proved in the inspiring paper of DinhSibony [6. Theorem 1] (cf. also [22]), assuming only $r(G)=n-1$. For non-abelian $G$, the finiteness of $N(G)$ is not true and we can at best expect that $N(G)$ is virtually included in $\operatorname{Aut}_{0}(X)$ (as done in Theorems 1.2 and 2.1), since a larger group $\widetilde{G}:=\operatorname{Aut}_{0}(X) G$ satisfies

$$
\begin{gathered}
\widetilde{G}\left|\mathrm{NS}_{\mathbb{C}}(X)=G\right| \mathrm{NS}_{\mathbb{C}}(X), N(\widetilde{G})=\operatorname{Aut}_{0}(X) \cdot N(G) \geq \operatorname{Aut}_{0}(X), \\
\widetilde{G} / N(\widetilde{G}) \cong G / N(G) .
\end{gathered}
$$

There are examples $(X, G)$ with $\operatorname{rank} r(G)=\operatorname{dim} X-1$ and $X$ complex tori or their quotients (cf. [6, Example 4.5], 22, Example 1.7]).

The proof of Theorem 1.2 is much harder than that of Theorem 2.1 because of the presence of singularities on $X$.

The conditions (i) - (iii) in Theorem 1.2 are quite necessary in deducing $X \cong T / F$ as in Theorem 1.2(4). Indeed, if $X \cong T / F$ as in Theorem 1.2(4), then $X$ has only quotient singularities and $d K_{X} \sim 0$ (linear equivalence) with $d=|F|$, and we may even assume that $X$ has only canonical singularities if we replace $X$ by its global index- 1 cover; thus $X$ is a minimal variety. If the pair $(X, G)$ is not minimal so that there is a non-isomorphic $G_{1}$-equivariant birational morphism $X \rightarrow Y$ as in Section 2.5, then the exceptional locus of this morphism is $G_{1^{-}}$and hence $G$ periodic, contradicting the fact that the rank $r(G)=n-1$ (cf. the proof of Claim 2.20).

The first key step in proving Theorem 1.2 is the analysis of our quasi-nef sequence $L_{1} \ldots L_{k}(0<k<n)($ cf. [19, $\left.\S 2.2]\right)$ and we are able to show that $L_{i}$ can actually be taken to be nef, when the $\operatorname{rank} r(G)=n-1$ (cf. Lemma 2.9). The second key step is Theorem 2.2, where we split $G$ as $N(G) H$ such that $H \mid H^{1,1}(X)$ is free abelian. Thus we have a nef and big class $A$ as the sum of nef common eigenvectors of $H$, leading to the vanishing of $A^{n-i} \cdot c_{i}(X)$, where $c_{i}(X)(i=1,2)$ are Chern classes (cf. [16, pages 265-267]). The third key step is to use the minimality of $(X, G)$ and 
Kawamata's base point freeness result for the $\mathbb{R}$-divisor (cf. [2, Theorem 3.9.1], available only for the projective variety at the moment) to deduce the vanishing of $c_{2}(X)$ as a linear form. This vanishing does not directly follow from the vanishing of $A^{n-2} \cdot c_{2}(X)$ because $A$ may not be ample. Now Theorem 1.2 (4) follows from the vanishing of $c_{i}(X)(i=1,2)$ and the characterization of the torus quotient originally deduced from Yau's deep result (cf. [1])

Remark 1.3.

(1) When $\operatorname{dim} X=3$, condition (i) in Theorem 1.2 can be replaced by: (i)' $X$ has at worst canonical singularities (cf. the Proof of Lemma 2.19 and [16. Corollary on p. 266]).

(2) Theorems 1.2 and 2.1 are not true when $n:=\operatorname{dim} X=2$. We used $n \geq 3$ to deduce the vanishing of $c_{2}(X) \cdot A^{n-2}$ as commented above.

With Theorem 2.2 in mind, we ask:

Question 1.4. Suppose a group $G$ acts on a compact complex Kähler manifold (say a complex torus) such that the null set $N(G)$ is a subgroup of (and hence normal in) $G$ and the quotient $G / N(G)$ is a free abelian group. Under what condition can we write $G$ (or its finite-index subgroup) as $G=N(G) \rtimes H$ with $H \leq G$ a free abelian subgroup of $G$ ?

\section{Proof of The THEOREMS}

In this section, we prove Theorem 1.2 in the introduction and the three results below.

When the $X$ below is non-algebraic, we don't require the minimality of the pair $(X, G)$.

Theorem 2.1. Let $X$ be an $n$-dimensional $(n \geq 3)$ compact complex Kähler manifold which is not algebraic. Let $G \leq \operatorname{Aut}(X)$ be a subgroup such that the induced action $G \mid H^{1,1}(X) \leq \operatorname{Aut}\left(H^{1,1}(X)\right)$ is solvable and $Z$-connected and that the rank $r(G)=n-1$ (i.e., $\left.G / N(G)=\mathbb{Z}^{\oplus n-1}\right)$. Assume that $X$ is minimal, i.e., the canonical divisor $K_{X}$ is contained in the closure of the Kähler cone of $X$. Then the following four assertions hold:

(1) The induced action $N(G) \mid H^{1,1}(X)$ is a finite group.

(2) $G \mid H^{1,1}(X)$ is a virtually free abelian group of rank $n-1$.

(3) Either $N(G)$ is a finite subgroup of $G$ and hence $G$ is a virtually free abelian group of rank $n-1$, or $X$ is a complex torus and the group $N(G) \cap \operatorname{Aut}_{0}(X)$ has finite-index in $N(G)$ and is Zariski-dense in $\operatorname{Aut}_{0}(X)(\cong X)$.

(4) We have $X \cong T / F$ for a finite group $F$ acting freely outside a finite set of a complex torus $T$. Further, for some finite-index subgroup $G_{1}$ of $G$, the action of $G_{1}$ on $X$ lifts to an action of $G_{1}$ on $T$.

The $X$ or the pair $(X, G)$ below is not assumed to be minimal.

Theorem 2.2. Let $X$ be an $n$-dimensional $(n \geq 2)$ compact complex Kähler manifold and $G \leq \operatorname{Aut}(X)$ a subgroup such that the induced action $G \mid H^{1,1}(X) \leq$ $\operatorname{Aut}\left(H^{1,1}(X)\right)$ is solvable and $Z$-connected and that the rank $r(G)=n-1$ (i.e., $\left.G / N(G)=\mathbb{Z}^{\oplus n-1}\right)$. Then, replacing $G$ by its finite-index subgroup, we can find a 
subgroup $H \leq G$ such that:

(1) $G=N(G) H$;

(2) $G \mid H^{1,1}(X)=\left(N(G) \mid H^{1,1}(X)\right) \rtimes\left(H \mid H^{1,1}(X)\right)$;

(3) $N(G) \mid H^{1,1}(X)$ is unipotent; and

(4) the induced action $H \mid H^{1,1}(X) \leq \operatorname{Aut}\left(H^{1,1}(X)\right)$ is a free abelian group of rank $n-1$, i.e., $H \mid H^{1,1}(X) \cong \mathbb{Z}^{\oplus n-1}$.

In the process of proving Theorem 2.2, we also deduce:

Proposition 2.3. For the $X$ and $G$ in Theorem 2.2, replacing $G$ by its finite-index subgroup, we can find some $g_{0} \in G \backslash N(G)$, such that the first dynamical degrees satisfy

$$
d_{1}(g)=d_{1}\left(g_{0}\right)^{t}
$$

for every $g \in G$ with $t \in \mathbb{Z}_{\geq 0}$ depending on $g$.

Remark 2.4. (1) In Theorem 2.2 and Proposition 2.3. if $X$ is a normal projective variety, then a similar proof implies the same conclusions but with all the $H^{1,1}(X)$ in Theorem 2.2 replaced by $\mathrm{NS}_{\mathbb{C}}(X)$.

(2) To allow singularities of $X$ in Theorem 2.1, we need the Kähler version of the birational contraction theorem [2, Theorem 3.9.1] and Miyaoka's pseudo-effectivity of $c_{2}(X)$ for minimal variety $X$, both of which seem to be very hard to confirm, since the Minimal Model Program has not been fully developed for Kähler manifolds.

2.5. s-cycles and minimal pairs. Let $X$ be an $n$-dimensional compact Kähler manifold or a normal projective variety. When $X$ is projective, set $\mathrm{NS}_{\mathbb{R}}(X):=$ $\mathrm{NS}(X) \otimes_{\mathbb{Z}} \mathbb{R}$. A codimension-s (i.e., dimension- $(n-s)$ ) cycle $D$ is an element in $H^{s, s}(X, \mathbb{R}):=H^{s, s}(X) \cap H^{2 s}(X, \mathbb{R})$ (resp. a linear combination of $(n-s)$ dimensional subvarieties with coefficients in $\mathbb{R}$ ) when $X$ is Kähler (resp. projective). Two codimension-s cycles $D_{i}$ are numerically equivalent, denoted as $D_{1} \equiv D_{2}$, if $\left(D_{1}-D_{2}\right) \cdot L_{1} \ldots L_{n-s}=0$ for all $L_{i}$ in $H^{1,1}(X, \mathbb{R})$ (resp. in $\left.\operatorname{NS}_{\mathbb{R}}(X)\right)$, where we use $D . L$ to denote the cup product (resp. intersection) for Kähler (resp. projective) $X$. Denote by $[D]$ the numerical equivalence class containing $D$ and

$$
N^{s}(X):=\{[D] ; D \text { is a codimension-s cycle }\}
$$

which is a finite-dimensional $\mathbb{R}$-vector space. We will loosely write $D \in N^{s}(X)$ by abuse of notation. Note that $N^{1}(X)=\mathrm{NS}_{\mathbb{R}}(X)$ when $X$ is projective. Denote by $K(X)$ (resp. $\operatorname{Amp}(X))$ the open Kähler (resp. ample) cone and $\overline{K(X)}$ (resp. $\operatorname{Nef}(X))$ its closure in $H^{1,1}(X, \mathbb{R})$ (resp. in $\mathrm{NS}_{\mathbb{R}}(X)$ ). Elements in $\overline{K(X)}$ and $\operatorname{Nef}(X)$ are called nef.

Let $X$ be a normal projective variety with at worst canonical singularities (cf. [10, Definition 2.34]) and $G \leq \operatorname{Aut}(X)$ a subgroup such that the null set $N(G)$ is a subgroup (and hence normal in $G$ ). The pair $(X, G)$ is non-minimal if: there are a finite-index subgroup $G_{1}$ of $G$ and a non-isomorphic $G_{1}$-equivariant birational morphism $X \rightarrow Y$ onto a normal projective variety $Y$ with at worst isolated canonical singularities. The pair $(X, G)$ is minimal if it is not non-minimal.

Lemma 2.6. Let $G$ be a group and $H \triangleleft G$ a finite normal subgroup. Suppose that

$$
G / H=\left\langle\bar{g}_{1}\right\rangle \times \cdots \times\left\langle\bar{g}_{r}\right\rangle \cong \mathbb{Z}^{\oplus r}
$$

for some $r \geq 1$ and $g_{i} \in G$. Then there is an integer $s>0$ such that $G_{1}:=$ $\left\langle g_{1}^{s}, \ldots, g_{r}^{s}\right\rangle$ satisfies

$$
G_{1}=\left\langle g_{1}^{s}\right\rangle \times \cdots \times\left\langle g_{r}^{s}\right\rangle \cong \mathbb{Z}^{\oplus r}
$$


and it is a finite-index subgroup of $G$; further, the quotient map $\gamma: G \rightarrow G / H$ restricts to an isomorphism $\gamma \mid G_{1}: G_{1} \rightarrow \gamma\left(G_{1}\right)$ onto a finite-index subgroup of $G / H$

Proof. We only need to find $s>0$ such that $g_{i}^{s}$ and $g_{j}^{s}$ are commutative to each other for all $i, j$. Since $G / H$ is abelian, the commutator subgroup $[G, G] \leq H$. Thus the commutators $\left[g_{1}^{t}, g_{2}\right](t>0)$ all belong to $H$. The finiteness of $H$ implies that $\left[g_{1}^{t_{1}}, g_{2}\right]=\left[g_{1}^{t_{2}}, g_{2}\right]$ for some $t_{2}>t_{1}$, which implies that $g_{1}^{s_{12}}$ commutes with $g_{2}$, where $s_{12}:=t_{2}-t_{1}$. Similarly, we can find an integer $s_{1 j}>0$ such that $g_{1}^{s_{1 j}}$ commutes with $g_{j}$. Set $s_{1}:=s_{12} \times \cdots \times s_{1 r}>0$. Then $g_{1}^{s_{1}}$ commutes with every $g_{j}$. Similarly, for each $i$, we can find an integer $s_{i}>0$ such that $g_{i}^{s_{i}}$ commutes with $g_{j}$ for all $j$. Now $s:=s_{1} \times \cdots \times s_{r}>0$ will do the job. This proves the lemma.

From now on until Section 2.14, we prove Theorem 2.2 and Proposition 2.3 ,

2.7. By [19, Proof of Theorem 1.2, §2.2], there is a quasi-nef sequence $L_{1} \ldots L_{k} \in$ $H^{k, k}(X)(1 \leq k \leq n-1)$ which is non-zero in $N^{k}(X)$, such that

$$
g^{*}\left(L_{1} \ldots L_{k}\right)=\chi_{1}(g) \cdots \chi_{k}(g)\left(L_{1} \ldots L_{k}\right)
$$

for all $k=1, \ldots, n-1$ with characters $\chi_{i}: G \rightarrow\left(\mathbb{R}_{>0}, \times\right)$, and that

$$
\begin{aligned}
\varphi: G & \rightarrow\left(\mathbb{R}^{n-1},+\right) \\
g & \mapsto\left(\log \chi_{1}(g), \ldots, \log \chi_{n-1}(g)\right)
\end{aligned}
$$

is a homomorphism having

$$
\operatorname{Ker}(\varphi)=N(G)
$$

and the following discrete subgroup of $\mathbb{R}^{\oplus n-1}$ as its image (now with $r(G)=n-1$ ):

$$
\operatorname{Im}(\varphi)=\mathbb{Z}^{\oplus r(G)} .
$$

Lemma 2.8. Each projection $\operatorname{Im}\left(\log \chi_{i}\right) \subset \mathbb{R}(1 \leq i \leq n-1)$ is a free abelian group of rank one.

Proof. It suffices to show that $\operatorname{Im}\left(\log \chi_{i}\right) \cong \mathbb{Z}$ since $\operatorname{rank} \operatorname{Im}(\varphi)=n-1$. If rank $\operatorname{Im}\left(\log \chi_{i}\right) \geq 2$, then Kronecker's theorem implies the existence of a sequence $f_{t} \in G$ with $\chi_{i}\left(f_{t}\right)>1$ so that $\lim _{t \rightarrow \infty} \chi_{i}\left(f_{t}\right)=1$ (cf. 18, proof of Theorem 3.1]), which contradicts the discreteness of $\operatorname{Im}(\varphi)$ as proved in [19, Claim 2.9]. This proves the lemma.

By the above result, $\operatorname{Im}(\varphi)$ is the direct product of $n-1$ cyclic groups $\operatorname{Im}\left(\log \chi_{i}\right)$ which are the $\varphi$-images of $\left\langle g_{i}\right\rangle$ say, with

$$
\lambda_{i}:=\chi_{i}\left(g_{i}\right)>1, \quad \chi_{j}\left(g_{i}\right)=1(j \neq i) .
$$

Hence

$$
G / N(G)=\left\langle\overline{g_{1}}, \ldots, \overline{g_{n-1}}\right\rangle=\left\langle\overline{g_{1}}\right\rangle \oplus \cdots \oplus\left\langle\overline{g_{n-1}}\right\rangle \cong \mathbb{Z}^{\oplus n-1}
$$

and

$$
\operatorname{Im}(\varphi)=\left\langle\varphi\left(g_{1}\right), \ldots, \varphi\left(g_{n-1}\right)\right\rangle=\left\langle\varphi\left(g_{1}\right)\right\rangle \oplus \cdots \oplus\left\langle\varphi\left(g_{n-1}\right)\right\rangle \cong \mathbb{Z}^{\oplus n-1} .
$$

By the generalization of the Perron-Frobenius theorem [3] applied to the action of $g_{i}^{ \pm 1}$ on the closure $\overline{K(X)}$ of the Kähler cone $K(X)$ (which spans $H^{1,1}(X, \mathbb{R})$ ), 
there are non-zero nef $L_{g_{i}^{ \pm 1}} \in \overline{K(X)}$ (which we now fix) such that

$$
\left(g_{i}^{ \pm 1}\right)^{*} L_{g_{i}^{ \pm 1}}=d_{1}\left(g_{i}^{ \pm 1}\right) L_{g_{i}^{ \pm 1}} .
$$

Note that the first dynamical degree $d_{1}\left(g_{i}^{ \pm 1}\right)>1$ since $g_{i}^{ \pm 1} \notin N(G)$.

Lemma 2.9. Let $k \in\{1, \ldots, n-1\}$. Let $M \in \overline{K(X)}$ be a non-zero nef element such that $g_{k}^{*} M=\lambda M$ for some $\lambda \neq 1$ (e.g., we can take $M=L_{g_{k}}, L_{g_{k}^{-1}}$ ).

(0) For $D\left(\right.$ e.g., $\left.D=L_{1} \ldots L_{k}\right)$ in $\overline{P^{k}(X)}$ (the closure of Kähler $(k, k)$-forms as defined in [12, before Lemma A.3]), $D=0$ in $N^{k}(X)$ if and only if $D=0$ in $H^{k, k}(X)$.

(1) Suppose that $\lambda \neq 1 / \lambda_{k}$ (e.g., we can take $\left.M=L_{g_{k}}\right)$. Then $L_{1} \ldots L_{k} \cdot M=0$ in $H^{k+1}(X)$.

(2) Suppose that $k \geq 2$. Then $L_{1} \ldots L_{k-1} . M$ is non-zero in $N^{k}(X)$.

(3) Suppose that $k=1$ and $\lambda>1$ (e.g., $\left.M=L_{g_{1}}\right)$. Then $M$ is parallel to $L_{1}$ in $H^{1,1}(X)$ and hence $\lambda=\lambda_{1}$. In particular, $L_{g_{1}}$ is parallel to $L_{1}$ in $H^{1,1}(X)$.

(4) Suppose that $k \geq 2$ and $\lambda \neq 1 / \lambda_{k}$. Then $L_{1} \ldots L_{k}$ and $L_{1} \ldots L_{k-1}$.M are parallel in $N^{k}(X)$. In particular, $\lambda=\lambda_{k}$.

(5) For all $1 \leq s \leq n-1, L_{1} \ldots L_{s}$ and $L_{g_{1}} \ldots L_{g_{s}}$ are parallel in $N^{s}(X)$.

(6) $\lambda$ equals either $\lambda_{k}>1$ or $1 / \lambda_{k}<1$.

(7) For all $1 \leq s \leq n-1$, we have

$$
d_{1}\left(g_{s}\right)=\lambda_{s}\left(=\chi_{s}\left(g_{s}\right)\right), \quad d_{1}\left(g_{s}^{-1}\right)=d_{1}\left(g_{s}\right)(>1) .
$$

(8) For all $1 \leq s \leq n-1, L_{1} \ldots L_{n-1} \cdot L_{g_{s}^{-1}}$ is a non-zero (positive) scalar in $N^{n}(X)=H^{n, n}(X, \mathbb{R}) \cong \mathbb{R}$.

Proof. The assertion (0) is well known; see e.g. [12, Lemma A.4].

(1) Suppose to the contrary that $L_{1} \ldots L_{k} \cdot M$ is non-zero in $H^{k, k}(X)$ (i.e., in $N^{k+1}(X)$ by the assertion $\left.(0)\right)$. Since

$$
g_{k}^{*}\left(L_{1} \ldots L_{k} \cdot L_{k+1}\right)=\lambda_{k}\left(L_{1} \ldots L_{k} \cdot L_{k+1}\right), \quad g_{k}^{*}\left(L_{1} \ldots L_{k} \cdot M\right)=\lambda_{k} \lambda\left(L_{1} \ldots L_{k} \cdot M\right)
$$

with $\lambda_{k} \neq \lambda_{k} \lambda$, we have $L_{1} \ldots L_{k+1} . L_{g_{k}} \neq 0$ in $N^{k+1}(X)$ (cf. [19, Lemma 2.3], [6. Lemma 4.4]). Inductively, the same $g_{k}^{*}$ action and [ibid.] imply that $L_{1} \ldots L_{t} . M$ is non-zero in $N^{t+1}(X)$ for all $k \leq t \leq n-1$. In particular, $L_{1} \ldots L_{n-1} . M$ equals a positive scalar $b_{n}$ in $N^{n}(X) \cong \mathbb{R}$. Thus $b_{n}=g_{k}^{*} b_{n}=g_{k}^{*}\left(L_{1} \ldots L_{n-1} \cdot M\right)=\lambda_{k} \lambda b_{n} \neq$ $b_{n}$, by the condition on $\lambda$. This is a contradiction.

(2) Since $g_{k}^{*} M=\lambda M$ and $g_{k}^{*} L_{1}=L_{1}$ with $\lambda \neq 1$, we have $L_{1} \cdot M \neq 0$ in $N^{2}(X)$ by [. Lemma 4.4].

Let $k \geq 3$ and let $t \geq 1$ be the largest integer such that $L_{1} \ldots L_{t} . M \neq 0$ in $N^{t+1}(X)$. If $t \geq k-1$, then (2) is true. Suppose to the contrary that $t \leq k-2$. Since

$$
g_{k}^{*}\left(L_{1} \ldots L_{t} \cdot L_{t+1}\right)=L_{1} \ldots L_{t} \cdot L_{t+1}, \quad g_{k}^{*}\left(L_{1} \ldots L_{t} \cdot M\right)=\lambda\left(L_{1} \ldots L_{t} \cdot M\right)
$$

with $\lambda \neq 1$, we have $L_{1} \ldots L_{t} \cdot L_{t+1} \cdot M \neq 0$ in $N^{t+2}(X)$ (cf. [19, Lemma 2.3], [6. Lemma 4.4]). This contradicts the maximality of $t$. So $t \geq k-1$. Hence (2) is true.

(3) By (1), $L_{1} \cdot L_{g_{1}}=0$ in $H^{1,1}(X)$, so $L_{1}$ and $M$ are parallel in $H^{1,1}(X)$ (cf. [6. Corollary 3.2]).

(4) We have $L_{1} \ldots L_{k} \cdot M=0$ by (1). Then (4) follows (cf. [19, Lemma 2.3], [6. Corollary 3.5]). Indeed, for the last part, just apply $g_{k}^{*}$ to the equality of the first part (cf. (2)). 
(5) follows from (3) and (4) with $M=L_{g_{k}}$, by induction on $k$.

(6) By (2), $L_{1} \ldots L_{k-1} . M \neq 0$ in $N^{k}(X)$. Suppose to the contrary that $\lambda \neq \lambda_{k}^{ \pm}$. Since

$$
g_{k}^{*}\left(L_{1} \ldots L_{k-1} \cdot L_{k}\right)=\lambda_{k}\left(L_{1} \ldots L_{k}\right), \quad g_{k}^{*}\left(L_{1} \ldots L_{k-1} \cdot M\right)=\lambda\left(L_{1} \ldots L_{k-1} \cdot M\right)
$$

with $\lambda_{k} \neq \lambda$, we have $L_{1} \ldots L_{k} \cdot M \neq 0$ in $N^{k+1}(X)$ (cf. [19, Lemma 2.3], 6, Lemma 4.4]), contradicting (1).

(7) follows from (6) with $M=L_{g_{k}}, L_{g_{k}^{-1}}$.

(8) Set $M:=L_{g_{k}^{-1}}$. By (2), we have the non-vanishing of $L_{1} \ldots L_{k-1} \cdot M$ in $N^{k}(X)$. As in the proof of (1), inductively, the action of $g_{k}^{*}$ implies the nonvanishing of $L_{1} \ldots L_{t} . M$ in $N^{t+1}(X)$ for all $k-1 \leq t \leq n-1$.

By Lemma 2.9, we may and will take

$$
L_{i}=L_{g_{i}} .
$$

Lemma 2.10. For all $1 \leq k \leq n-1$, as elements in $H^{2,2}(X)$, we have

$$
L_{g_{k}}^{2}=0, L_{g_{k}^{-1}}^{2}=0 .
$$

In particular, in $H^{1,1}(X)$, when $g_{k}^{*} M=\lambda M$ for some non-zero nef $M$, then $\lambda=$ $d_{1}\left(g_{k}\right)$ and $M$ is parallel to $L_{g_{k}}$ if $\lambda>1$, and $\lambda^{-1}=d_{1}\left(g_{k}\right)=d_{1}\left(g_{k}^{-1}\right)$ and $M$ is parallel to $L_{g_{k}^{-1}}$ if $\lambda<1$. So the choice of nef $L_{g_{k}}$ (resp. $\left.L_{g_{k}^{-1}}\right)$ is unique, up to a positive multiple.

Proof. We take $L_{i}=L_{g_{i}}$. Set $M=L_{g_{k}}$ or $L_{g_{k}^{-1}}$.

We claim that $L_{1} \ldots L_{k-1} \cdot M^{2}$ vanishes in $N^{k+1}(X)$ (i.e., in $H^{k+1, k+1}(X)$, by Lemma 2.9 (0)). When $M=L_{g_{k}}$, this is true by Lemma 2.9)(1). When $M=L_{g_{k}^{-1}}$, suppose to the contrary that $L_{1} \ldots L_{k-1} \cdot M^{2}$ is non-vanishing in $N^{k+1}(X)$. Note that $L_{1} \ldots L_{k} . M \neq 0$ in $N^{k+1}(X)$ by Lemma 2.9(8). Since

$$
\begin{aligned}
& g_{k}^{*}\left(L_{1} \ldots L_{k-1} \cdot M \cdot M\right)=\left(1 / \lambda_{k}\right)^{2}\left(L_{1} \ldots L_{k-1} \cdot M \cdot M\right), \\
& g_{k}^{*}\left(L_{1} \ldots L_{k-1} \cdot M \cdot L_{k}\right)=\left(1 / \lambda_{k}\right) \lambda_{k}\left(L_{1} \ldots L_{t-1} \cdot L_{k} \cdot L_{t}\right)
\end{aligned}
$$

with $\left(1 / \lambda_{k}\right)^{2} \neq\left(1 / \lambda_{k}\right) \lambda_{k}$, we have the non-vanishing of $L_{1} \ldots L_{k} \cdot M^{2}$ in $N^{k+2}(X)$ (cf. [19, Lemma 2.3], 6, Lemma 4.4]). Inductively, the action of $g_{k}^{*}$ and [ibid.] imply the non-vanishing of $b_{t+2}:=L_{1} \ldots L_{t} \cdot M^{2}$ in $N^{t+2}(X)$, for all $k-1 \leq t \leq n-2$. Thus $b_{n}=g_{k}^{*} b_{n}=\alpha\left(1 / \lambda_{k}\right)^{2} b_{n} \neq b_{n}$, where $\alpha=1$ (when $k=n-1$ ) or $\alpha=\lambda_{k}$ (when $k \leq n-2$ ), a contradiction! Hence the claim is true. In particular, the lemma is true when $k=1$.

Let $k \geq 2$. Suppose to the contrary that $M^{2}$ is non-zero in $H^{2,2}(X)$ (i.e., in $N^{2}(X)$ by Lemma[2.9 (0)). By the claim above, we can choose $1 \leq t \leq k-1$ to be the smallest integer such that $L_{1} \ldots L_{t} \cdot M^{2}=0$ in $N^{t+2}(X)$. Thus $L_{1} \ldots L_{t-1} \cdot M^{2} \neq 0$ in $N^{t+1}(X)$. Note that $L_{1} \ldots L_{t} \cdot M \neq 0$ in $N^{t+1}(X)$, by Lemma 2.9)(8) (for $M=$ $L_{g_{k}^{-1}}$ ) and by the non-vanishing of $L_{1} \ldots L_{n-1}$ in $N^{n-1}(X)$ (for $M=L_{k}$ ). Since

$$
\begin{aligned}
& g_{k}^{*}\left(L_{1} \ldots L_{t-1} \cdot M \cdot M\right)=\lambda^{2}\left(L_{1} \ldots L_{t-1} \cdot M \cdot M\right), \\
& g_{k}^{*}\left(L_{1} \ldots L_{t-1} \cdot M \cdot L_{t}\right)=\lambda\left(L_{1} \ldots L_{t-1} \cdot M \cdot L_{t}\right)
\end{aligned}
$$

with $\lambda^{2} \neq \lambda$ (because $\lambda=\lambda_{k}$ or $\lambda_{k}^{-1}$ for $M=L_{g_{k}}$ or $L_{g_{k}^{-1}}$ ), we have the nonvanishing of $L_{1} \ldots L_{t} \cdot M^{2}$ in $N^{t+2}(X)$ (cf. [19, Lemma 2.3], [6, Lemma 4.4]). This contradicts the choice of $t$. 
For the final part, we may suppose that $\lambda \neq 1$. By Lemma 2.9, if $\lambda>1$ (resp. $\lambda<1)$, we have $\lambda=d_{1}\left(g_{k}\right)=d_{1}\left(g_{k}^{-1}\right)\left(\right.$ resp. $\left.\lambda^{-1}=d_{1}\left(g_{k}\right)=d_{1}\left(g_{k}^{-1}\right)\right)$. Taking $M$ or $M+L_{g_{k}}$ (resp. $M$ or $M+L_{g_{k}^{-1}}$ ) as new $L_{g_{k}}$ (resp. $L_{g_{k}^{-1}}$ ), the first part shows that, in $H^{2,2}(X)$, we have $M^{2} \stackrel{L_{g_{k}}}{=} L_{\left(M+L_{g_{k}}\right.}^{2}=0$ (resp. $M^{2}=L_{g_{k}^{-1}}^{2}=$ $\left.\left(M+L_{g_{k}^{-1}}\right)^{2}=0\right)$, hence $M \cdot L_{g_{k}}=0$ (resp. $\left.M \cdot L_{g_{k}^{-1}}=0\right)$. Then $M$ and $L_{g_{k}}$ (resp. $M$ and $L_{g_{k}^{-1}}$ ) are parallel in $H^{1,1}(X)$, by [6, Corollary 3.2].

Set $L_{n}=L_{g_{k}^{-1}}$ for some $1 \leq k \leq n-1$. Then

$$
A:=L_{1}+\cdots+L_{n}
$$

is a nef and big class because $H^{n} \geq L_{1} \ldots L_{n-1} \cdot L_{n}>0$ by Lemma 2.9)(8). We may also write $g^{*}\left(L_{1} \ldots L_{n}\right)=\chi_{1}(g) \cdots \chi_{n}(g)\left(L_{1} \ldots L_{n}\right)$. Then

$$
\chi_{1} \ldots \chi_{n}=1
$$

since $L_{1} \ldots L_{n}$ is a non-zero (positive) scalar in $N^{n}(X) \cong \mathbb{R}$.

Lemma 2.11. $C:=\left(Z(G) \mid H^{1,1}(X)\right) \cap\left(N(G) \mid H^{1,1}(X)\right)$ is a finite subgroup of $\operatorname{Aut}\left(H^{1,1}(X)\right)$.

Proof. Take any $z \mid H^{1,1}(X) \in C$. Then $z\left|H^{1,1}(X) \in Z(G)\right| H^{1,1}(X)$, and hence $z \mid H^{1,1}(X)$ commutes with every $g_{i} \mid H^{1,1}(X)$. Thus, $g_{i}^{*} z^{*} L_{g_{i}}=z^{*} g_{i}^{*} L_{g_{i}}=$ $d_{1}\left(g_{i}\right) z^{*} L_{g_{i}}$, so $z^{*} L_{g_{i}}$ and $L_{g_{i}}$ are parallel by Lemma 2.10, and hence are equal, since $z \in N(G)$ has the first dynamical degree $d_{1}(z)=1$. By the same reasoning, $z^{*} L_{g_{i}^{-1}}=L_{g_{i}^{-1}}$. Thus $z^{*}$ fixes the nef and big class $A=\sum_{i=1}^{n} L_{i}$ with $L_{i}=L_{g_{i}}$ and $L_{n}=L_{g_{1}^{-1}}$. Hence $z^{s} \in \operatorname{Aut}_{0}(X)$ for some $s>0$ by [11, Proposition 2.2] (cf. [20. Lemma 2.23] and note that a big class is the sum of a Kähler class and a positive real current, according to Demailly-Paun). Thus $z^{s}$ acts trivially on the lattice $H^{2}(X, \mathbb{Z})$ and hence also on $H^{1,1}(X)$. So $C$ is a periodic group and defined over $\mathbb{Z}$, and hence is a finite group by Burnside's theorem (cf. 14, Proof of Proposition $2.2])$. This proves the lemma.

Now Theorem 2.2 follows from Proposition 2.12

Proposition 2.12. Replacing $G$ by its finite-index subgroup and $g_{i}$ by some element in $g_{i} N(G)$, the group $H:=\left\langle g_{1}, \ldots, g_{n-1}\right\rangle$ has its image $H \mid H^{1,1}(X)$ in $\operatorname{Aut}\left(H^{1,1}(X)\right)$, a free abelian group of rank $n-1$, so that

$$
G=N(G) H, N(G) \mid H^{1,1}(X)
$$

is unipotent, and

$$
G \mid H^{1,1}(X)=\left(N(G) \mid H^{1,1}(X)\right) \rtimes\left(H \mid H^{1,1}(X)\right) .
$$

Proof. By [17, Proposition 4.1] and Selberg's lemma, replacing $G$ by its finiteindex subgroup, we may assume that $G \mid H^{1,1}(X)$ is torsion free. Note that the set $U(G):=\left\{u \in G ; u \mid H^{1,1}(X)\right.$ is unipotent $\}$ is a finite-index subgroup of $N(G)$ and a characteristic subgroup of $G$ (cf. [14, Proof of Prop. 2.2], 44, Theorem 3.1]). Replacing $G$ by a finite-index subgroup of $G_{1}:=U(G)\left\langle g_{1}, \ldots, g_{n-1}\right\rangle$, we may assume that $N(G)=U(G)$ (applying Lemma 2.6 to the group $G_{1} / U(G)$ ). Since $\operatorname{Ker}\left(G \rightarrow G \mid H^{1,1}(X)\right) \leq N(G)$, we get

$$
\left(G \mid H^{1,1}(X)\right) /\left(N(G) \mid H^{1,1}(X)\right) \cong G / N(G)=\left\langle\overline{g_{1}}, \ldots, \overline{g_{n-1}}\right\rangle \cong \mathbb{Z}^{\oplus n-1},
$$


which is abelian and hence nilpotent. By [15, Thm. 3, p. 48], there is a subgroup $H$ of $G$ such that $H \mid H^{1,1}(X)$ is nilpotent and $G=N(G) H$, after replacing $G$ by its finite-index subgroup. Replacing $g_{i}$ by some element in $g_{i} N(G)$, we may assume that $g_{i} \in H$.

Suppose $H \mid H^{1,1}(X)$ is non-abelian. Then the commutator subgroup $[H, H] \mid$ $H^{1,1}(X)$ is non-trivial and is contained in $[G, G] \mid H^{1,1}(X)$, while the latter is contained in the group $N(G) \mid H^{1,1}(X)$ because $G / N(G)$ is abelian. Since $H \mid H^{1,1}(X)$ is nilpotent, its centre $\left(\leq[H, H]\left|H^{1,1}(X) \leq N(G)\right| H^{1,1}(X)\right)$ contains a non-trivial element $z \mid H^{1,1}(X)$ (of course commutative with all $g_{i} \mid H^{1,1}(X)$ ). The proof of Lemma 2.11 shows that $z\left|H^{1,1}(X) \in G\right| H^{1,1}(X)$ is of finite order, contradicting the torsion freeness assumption of $G \mid H^{1,1}(X)$.

Thus, we may assume that $H \mid H^{1,1}(X)$ is abelian (and free because $G \mid H^{1,1}(X)$ is as well). Replacing $H$ by $\left\langle g_{1}, \ldots, g_{n-1}\right\rangle$, we may assume that $H \mid H^{1,1}(X)$ is free abelian and of rank $n-1$ because it is generated by $n-1$ elements and dominates $\mathbb{Z}^{\oplus n-1}$ via the surjective composite below (cf. the display (*) above):

$$
\begin{aligned}
H \mid H^{1,1}(X) & \rightarrow\left(H \mid H^{1,1}(X)\right) /\left(H \mid H^{1,1}(X)\right) \cap\left(N(G) \mid H^{1,1}(X)\right) \\
& \rightarrow\left(G \mid H^{1,1}(X)\right) /\left(N(G) \mid H^{1,1}(X)\right) \cong\left\langle\overline{g_{1}}, \ldots, \overline{g_{n-1}}\right\rangle \cong \mathbb{Z}^{\oplus n-1} .
\end{aligned}
$$

Now the same dominance (between free abelian groups of the same rank) implies that $\left(H \mid H^{1,1}(X)\right) \cap\left(N(G) \mid H^{1,1}(X)\right)=\{$ id $\}$ (or else $\mathbb{Z}^{\oplus n-1}$ is dominated by a free abelian group, a quotient of $H$, of rank $\leq n-2$, absurd!). Thus the display of and hence the entire proposition follow.

Lemma 2.13. $L_{g_{s}^{-1}}(1 \leq s \leq n-1)$ are all parallel to $L_{g_{1}^{-1}}$ in $H^{1,1}(X)$.

Proof. Since $L_{g_{i}^{-1}}$ is unique up to a multiple (cf. Lemma 2.10) and is parallel to $L_{g_{i}^{-t}}$ for any $t>0$, we may assume that $G=N(G) H$ as in Proposition 2.12. Since $g_{i}\left|H^{1,1}(X) \in H\right| H^{1,1}(X)(1 \leq i \leq n-1)$ are commutative to each other, we may assume that both $L_{g_{i}}$ and $L_{g_{i}^{-1}}$ are common nef eigenvectors of $H$. Set $L:=L_{g_{1}^{-1}}$ and write $g_{s}^{*} L=\mu_{s} L$ with $\mu_{1}=1 / d_{1}\left(g_{1}^{-1}\right)=1 / d_{1}\left(g_{1}\right)=1 / \lambda_{1}$ (cf. Lemma 2.9). Applying $g_{s}^{*}$ to the non-zero scalar $L_{g_{1}} \ldots L_{g_{n-1}} . L$ in $N^{n}(X) \cong \mathbb{R}$ in Lemma 2.9. we get

$$
L_{g_{1}} \ldots L_{g_{n-1}} \cdot L=\left(d_{1}\left(g_{s}\right) \mu_{s}\right)\left(L_{g_{1}} \ldots L_{g_{n-1}} \cdot L\right) .
$$

Hence $1=d_{1}\left(g_{s}\right) \mu_{s}$ and $\mu_{s}=1 / d_{1}\left(g_{s}\right)=1 / d_{1}\left(g_{s}^{-1}\right)$. Thus $\left(g_{s}^{-1}\right)^{*} L=d_{1}\left(g_{s}^{-1}\right) L$ and the lemma follows (cf. Lemma 2.10).

2.14. Proof of Proposition 2.3. Since $G \mid H^{1,1}(X)$ is solvable and $Z$-connected and hence upper-triangularizable by the Lie-Kolchin theorem and by Proposition 2.12, we may assume that $G=H=\left\langle g_{1}, \ldots, g_{n-1}\right\rangle$. By Lemma 2.13, $L_{1}^{\prime}:=L_{g_{1}^{-1}}$ is a nef common eigenvector of $G$. As in [19, Proof of Theorem $1.1 ; \S 2.2]$, we may construct a quasi-nef sequence $L_{1}^{\prime} \ldots L_{k}^{\prime}(1 \leq k \leq n-1)$ so that $g^{*}\left(L_{1}^{\prime} \ldots L_{k}^{\prime}\right)=$ $\left(\chi_{1}^{\prime}(g) \ldots \chi_{k}^{\prime}(g)\right)\left(L_{1}^{\prime} \ldots L_{k}^{\prime}\right)$ and the homomorphism

$$
\begin{aligned}
\varphi^{\prime}: G & \rightarrow\left(\mathbb{R}^{\oplus n-1},+\right), \\
g & \mapsto\left(\log \chi_{1}^{\prime}(g), \ldots, \log \chi_{n-1}^{\prime}(g)\right)
\end{aligned}
$$

satisfies

$$
\operatorname{Ker}\left(\varphi^{\prime}\right)=N(G), \quad \operatorname{Im}\left(\varphi^{\prime}\right) \cong \mathbb{Z}^{\oplus n-1}
$$


As in the proof of Lemma 2.8, the projection $\operatorname{Im}\left(\log \chi_{1}^{\prime}\right) \cong \mathbb{Z}$ and hence is generated by some $\log \mu:=\log \chi_{1}^{\prime}\left(g_{b}\right)$. As in the proof of Lemma 2.13, $d_{1}\left(g_{s}\right) L_{1}^{\prime}=\left(g_{s}^{-1}\right)^{*} L_{1}^{\prime}=$ $\chi_{1}^{\prime}\left(g_{s}^{-1}\right) L_{1}^{\prime}=\mu^{t} L_{1}^{\prime}$ with $t \in \mathbb{Z}_{>0}$ depending on $g_{s}$. Hence $d_{1}\left(g_{s}\right)=d_{1}\left(g_{b}\right)^{t}$. Replacing $g_{i}$ 's by their powers, we may assume that $\lambda:=d_{1}\left(g_{i}\right)$ is independent of $i \in\{1, \ldots, n-1\}$.

For every $g \in G \backslash N(G)$, since $G \mid H^{1,1}(X)$ is now commutative, we may assume that $L_{g}$ is a nef common eigenvector of $G$. If $L_{g_{1}} \ldots L_{g_{n-1}} . L_{g} \neq 0$, then applying $g^{*}$ we get

$$
\chi_{1}(g) \ldots \chi_{n-1}(g) \cdot d_{1}(g)=1 .
$$

If $L_{g_{1}} \ldots L_{g_{n-1}} \cdot L_{g}=0$, then as in Lemma 2.9, 6, Lemma 4.4] implies that $d_{1}(g)=$ $\chi_{i}(g)$ for some $i$. Thus, $d_{1}(g)$ is either $\chi_{i}(g)$ or $\prod_{j=1}^{n-1} \chi_{j}\left(g^{-1}\right)$, which is an integer power of $\lambda$. If we express $g$ as a product of powers of $g_{i}$, use Lemma 2.9(7) and note that $\chi_{i}\left(g_{j}\right)=1(i \neq j)$. This proves Proposition 2.3.

2.15. With the notation and assumption of Theorem 1.2 suppose that $c_{2}(X) \neq 0$ as an element in $N^{n-2}(X)$, i.e., as a linear form on $N^{1}(X) \times \cdots \times N^{1}(X),(n-2)$ of them (cf. [16, pages 265-267], [22, Definition 2.4]). Since $X$ is minimal, Miyaoka's pseudo-effectivity of $c_{2}(X)$ implies that $c_{2}(X) \geq 0$ on $\operatorname{Nef}(X) \times \cdots \times \operatorname{Nef}(X),(n-2)$ of them (cf. [16, Theorem 4.1] and the references therein). Hence $c_{2}(X)>0$ on the self-product of the ample cone $\operatorname{Amp}(X) \times \cdots \times \operatorname{Amp}(X)$, since $N^{1}(X)$ is spanned by $\operatorname{Amp}(X)$, which is an open cone.

Since $c_{2}(X) \cdot \operatorname{Amp}(X)$ is a non-zero cone of $N^{3}(X)$ and is stable under the natural action of $G$, and since $G \mid N^{1}(X)$ is solvable and $Z$-connected, there are nef (and indeed ample) divisors $P(t)$ such that

$$
c_{2}(X) \cdot M_{1}:=\lim _{t \rightarrow \infty} c_{2}(X) \cdot P(t)
$$

is non-zero in $N^{3}(X)$ and a common $G$-eigenvector (cf. [9, Theorem 1.1]); note that $M_{1} \in N^{1}(X)$ may not be nef since the cone $c_{2}(X)$. Nef $(X)$ may not be closed in $N^{3}(X)$. By the same reasoning, we can construct $c_{2}(X) \cdot M_{1} \cdot M_{2}$. Continuing the process, we get:

Lemma 2.16. With the notation and assumption of Theorem 1.2 , suppose that $c_{2}(X) \neq 0$ in $N^{2}(X)$. Then we have:

(1) There is a sequence $0 \neq c_{2}(X) \cdot M_{1} \ldots M_{k} \in N^{k+2}(X)(1 \leq k \leq n-3)$ (called a pseudo-effective sequence) which is positive on the self-product of the ample cone $\operatorname{Amp}(X) \times \cdots \times \operatorname{Amp}(X)(n-k-2$ of them $)$, such that

$$
g^{*}\left(c_{2}(X) \cdot M_{1} \ldots M_{k}\right)=\chi_{1}^{\prime}(g) \ldots \chi_{k}^{\prime}(g)\left(c_{2}(X) \cdot M_{1} \ldots M_{k}\right)
$$

for all $k$ with characters $\chi_{i}^{\prime}: G \rightarrow\left(\mathbb{R}_{>0}, \times\right)$.

(2) In particular, $C:=c_{2}(X) \cdot M_{1} \ldots M_{n-3}$ is a non-zero element in the closed cone $\overline{\mathrm{NE}}(X)$ of effective 1-cycles (which is dual to the nef cone $\operatorname{Nef}(X)$ ).

2.17. Proof of Theorem 1.2. The proof will almost fill up the rest of the paper. We may and will freely replace $G$ by its finite-index subgroups. We may assume that $G=N(G) H$ with $H=\left\langle g_{1}, \ldots, g_{n-1}\right\rangle$ so that $H \mid \mathrm{NS}_{\mathbb{C}}(X) \cong \mathbb{Z}^{\oplus n-1}$ as in Theorem 2.2 and satisfies the four assertions there, but with $H^{1,1}(X)$ replaced by $\mathrm{NS}_{\mathbb{C}}(X)$ (cf. Remark 2.4). We use the notation in the proof of Theorem 2.2 and let

$$
A_{H}:=L_{g_{1}}+\cdots+L_{g_{n-1}}+L_{g_{1}^{-1}}
$$


be the nef and big divisor, where $L_{g_{j}^{ \pm}} \in \operatorname{Nef}(X)$ can be chosen to be common eigenvectors of $H$ since $H \mid \mathrm{NS}_{\mathbb{R}}(X)$ is commutative.

Lemma 2.18. (1) Let $D \in N^{s}(X)(0<s<n)$ such that $h^{*} D=D$ for all $h \in H$. Then D. $A_{H}^{n-s}=0$.

(2) In particular, for the Chern classes $c_{i}(X)(i=1,2)$, we have $c_{i}(X) \cdot A_{H}^{n-i}=$ 0 .

(3) Hence $K_{X} \sim_{\mathbb{Q}}$ 0, i.e., a positive multiple of $K_{X}$ is linearly equivalent to zero.

Proof. Take $M:=L_{1}^{i_{1}} \cdots L_{n}^{i_{n}}$ with $\sum_{k=1}^{n} i_{k}=n-s$. For $h \in H$, we have $h^{*} M=$ $e(h) M$ with $e(h)=\chi_{1}(h)^{i_{1}} \cdots \chi_{n}(h)^{i_{n}}$. Since $\mathbb{R} \ni M . D=h^{*} M . h^{*} D=e(h) M . D$ and since $A_{H}^{n-s}$ is a combination of such $M$, it suffices to show that $e(h) \neq 1$ for some $h \in H$ (so that $M . D=0$ ). Suppose to the contrary that $e(h)=1$ for all $h \in H$. Taking $\log$ and using $\chi_{1} \ldots \chi_{n}=1$, we have $\left(i_{1}-i_{n}\right) \log \chi_{1}+$ $\cdots+\left(i_{n-1}-i_{n}\right) \log \chi_{n-1}=0$ on $H$. Since the image of the homomorphism $\varphi=$ $\left(\log \chi_{1}, \ldots, \log \chi_{n-1}\right)$ is a spanning lattice in $\mathbb{R}^{\oplus n-1}$ of rank $n-1$ (cf. Section 2.7), this happens only when $i_{1}-i_{n}=\cdots=i_{n-1}-i_{n}=0$. Thus $n-1 \geq n-s=$ $\sum_{k=1}^{n} i_{k}=n i_{1}$, so $i_{1}=0$ and hence $s=n$. This is absurd.

Since $X$ is minimal and hence $K_{X}$ is nef, the vanishing of $K_{X} \cdot A_{H}^{n-1}$ and 13 , Lemma 2.2] imply that $K_{X} \equiv 0$ (numerically). Hence $K_{X} \sim_{\mathbb{Q}} 0$ (cf. [8, Theorem 8.2]). This proves Lemma 2.18 ,

Lemma 2.19. Theorem 1.2 is true when $c_{2}(X)=0$ in $N^{2}(X)$.

We prove the lemma. Set $A:=A_{H}$. The vanishing of $c_{2}(X)$ in $N^{2}(X)$ implies the vanishing of the orbifold second Chern class of $X$ (cf. [16, Proposition 1.1]). This and the vanishing of $c_{1}(X)$ in Lemma 2.18 imply the existence of a finite surjective morphism $T^{\prime} \rightarrow X$ from an abelian variety $T^{\prime}$, based on a deep result of S. T. Yau (cf. [1, 5, Theorem 7.6]). This is the place we need the singularities of $X$ to be of quotient type (cf. also Remark 1.3).

Since $K_{T^{\prime}} \sim 0 \sim_{\mathbb{Q}} K_{X}$, the map $T^{\prime} \rightarrow X$ is étale in codimenion one. Let $T \rightarrow X$ be the Galois cover corresponding to the unique maximal lattice $L$ in $\pi_{1}(X \backslash \operatorname{Sing} X)$ so that $T$ is an abelian variety. Then $X=T / F$ with

$$
F=\pi_{1}(X \backslash \operatorname{Sing} X) / L=\operatorname{Gal}(T / X),
$$

and there is an exact sequence

$$
1 \rightarrow F \rightarrow \widetilde{G} \stackrel{\gamma}{\rightarrow} G \rightarrow 1
$$

where $\widetilde{G}$ (the lifting of the original $G$ ) acts faithfully on $T$ (cf. 11, $\S 3$, especially the Proof of Prop. 3] applied to étale-in-codimension-one covers, and also [13, Prop. $3.5])$.

By [20, Lemma 2.6] or [12, Lemma A.8], $N(\widetilde{G})=\gamma^{-1}(N(G))$. Hence

$$
\widetilde{G} / N(\widetilde{G}) \cong G / N(G) \cong \mathbb{Z}^{\oplus n-1}, \quad r(\widetilde{G})=r(G)=n-1 .
$$

Since $N(\widetilde{G}) \mid \mathrm{NS}_{\mathbb{C}}(X)$ is virtually unipotent (cf. Proposition 2.12), we may assume that $\widetilde{G} \mid \mathrm{NS}_{\mathbb{C}}(X)$ is solvable and $Z$-connected after $G$ is replaced by its finite-index subgroup.

If Theorem $1.2(1)$ is not true, then $N(\widetilde{G}) \mid \mathrm{NS}_{\mathbb{R}}(T)$ is also an infinite group. Thus [22, Proof of Theorem 1.1(3), page 2338] shows the existence of a $\widetilde{G}$-equivariant 
fibration $T \rightarrow T / B$ with $0<B<T$ a subtorus of $T$ fixed by the unipotent elements of $\widetilde{G} \mid H^{1,1}(T)$. This leads to rank $r(G)=r(\widetilde{G}) \leq n-2$ (cf. [19, Proof of Lemma 2.10]), contradicting the assumption $r(G)=n-1$. Hence assertion (1) of Theorem 1.2 is true. (2) follows from (1) and Lemma 2.6.

For assertion (3), take an ample divisor (or a Kähler class for the purpose of Theorem 2.1) $M^{\prime}$ on $X$. Then $M:=\sum h_{t}^{*} M^{\prime}$, where $h_{t}$ runs in the finite group $N(G) \mid \mathrm{NS}_{\mathbb{R}}(X)$, is an ample divisor (or a Kähler class) and is stable under the action of $N(G)$. Hence $N(G) \leq \operatorname{Aut}_{M}(X):=\left\{f \in \operatorname{Aut}(X) \mid f^{*} M=M\right\}$, where $\left|\operatorname{Aut}_{M}(X): \operatorname{Aut}_{0}(X)\right|<\infty$ by [11, Proposition 2.2] or [7, Theorem 4.8]. Thus for

$$
N_{0}:=N(G) \cap \operatorname{Aut}_{0}(X)
$$

we have

$$
N(G) / N_{0} \cong\left(\operatorname{Aut}_{0}(X) \cdot N(G)\right) / \operatorname{Aut}_{0}(X) \leq \operatorname{Aut}_{M}(X) / \operatorname{Aut}_{0}(X),
$$

where the latter is a finite group. If $N_{0}$ is finite, then so is $N(G)$, and the first case of assertion (3) is true (cf. Lemma 2.6).

Suppose that $N_{0}$ is infinite. We shall show that the second case of assertion (3) occurs. First, $\operatorname{Aut}_{0}(X) \neq 1$. If the linear part of $\operatorname{Aut}_{0}(X)$ is non-trivial, then $X$ is ruled (and hence uniruled) (cf. [7, Proposition 5.10]), contradicting the assumption that $X$ is a minimal variety with only canonical singularities and hence non-uniruled by the well-known Miyaoka-Mori uniruledness criterion.

Therefore, the linear part $\operatorname{Aut}_{0}(X)$ is trivial. Then $\operatorname{Aut}_{0}(X)$ is a complex torus and (cf. [11, Theorem 3.12] or [7, Theorem 5.5])

$$
1 \leq \operatorname{dim} \operatorname{Aut}_{0}(X) \leq \operatorname{dim} \operatorname{Alb}(X)=q(X)
$$

where $\operatorname{Alb}(X)$ is the Albanese variety of $X$ and $q(X)=h^{1}\left(X, \mathcal{O}_{X}\right)$ is the irregularity of $X$. By [19, Lemma 2.13] applied to a resolution of $X$ and using [8, Lemma 8.1], the albanese map $\operatorname{alb}_{X}: X \rightarrow \operatorname{Alb}(X)$ is a birational and surjective morphism.

Claim 2.20. $X$ has no positive-dimensional subvariety $Y^{\prime}$ which is $G$-periodic (i.e., $Y^{\prime}$ is stabilized by a finite-index subgroup of $G$ ).

Proof. We prove the claim. We have already proved the finiteness of $N(\widetilde{G}) \mid \mathrm{NS}_{\mathbb{C}}(T)$. Replacing $\widetilde{G}$ by its finite-index subgroup and by Lemma 2.6 we may assume that $\widetilde{G} \mid \mathrm{NS}_{\mathbb{C}}(T)$ and even $\widetilde{G} /\left(\widetilde{G} \cap \operatorname{Aut}_{0}(T)\right)$ are free abelian groups of rank $n-1$.

Suppose a positive-dimensional subvariety $Y^{\prime} \subset X$ is $G$-periodic. Then a subvariety $Y \subset T$ (dominating $Y^{\prime}$ ) is $\widetilde{G}$-periodic and is even stabilized by $\widetilde{G}$ after this group is replaced by its finite-index subgroup. By the proof of [21, Lemma 2.11], there is a $\widetilde{G}$-equivariant homomorphism $T \rightarrow T / B$ with $\operatorname{dim}(T / B) \in\{1, \ldots, n-1\}$. Thus the $\operatorname{rank} r(\widetilde{G}) \leq \operatorname{dim} T-2=n-2($ cf. [19, Lemma 2.10]). This contradicts the fact that $r(\widetilde{G})=r(G)=n-1$. This proves Claim 2.20.

We return to the proof of Lemma 2.19

The action of (the original group) $G$ on $X$ induces an action of $G$ on the Albanese variety $\operatorname{Alb}(X)$ so that $\operatorname{alb}_{X}$ is $G$-equivariant, by the universal property of the albanese variety. If $\operatorname{alb}_{X}$ is not an isomorphism, then its exceptional locus (where the map is not isomorphic) is $G$-periodic and positive-dimensional by Zariski's main theorem, contradicting Claim 2.20. Thus alb . $_{X}$ is an isomorphism, and hence $X$ is an abelian variety. Since $r(G)=n-1$, [19, Lemma 2.14] implies that the Zariskiclosure $\overline{N_{0}}$ of $N_{0}$ in the translation group $\operatorname{Aut}_{0}(X) \cong X$ acts (as translations) on 
the torus $X$ with a Zariski-dense open orbit and is hence equal to $\operatorname{Aut}_{0}(X)$. So the second case of assertion (3) occurs.

For Theorem $1.2(4)$, since $F \triangleleft \widetilde{G}$, the fixed locus

$$
T^{F}=\{t \in T \mid f(t)=t \text { for some id } \neq f \in F\}
$$

is $\widetilde{G}$-stable. If this locus is positive-dimensional, then its image in $X$ is $G$-periodic, contradicting Claim 2.20. If $X$ is not yet an abelian variety, then by the proved assertion $(3), N(\widetilde{G})=\gamma^{-1}(N(G))$ is finite. Applying Lemma 2.6 to

$$
(\widetilde{G} / F) /(N(\widetilde{G}) / F) \cong G / N(G) \cong \mathbb{Z}^{\oplus n-1}
$$

there is a rank $n-1$, free abelian, finite-index subgroup $\widetilde{G_{1}} / F$ of $\widetilde{G} / F=G$. Applying Lemma 2.6 to $\widetilde{G_{1}} / F$, our $\gamma: \widetilde{G} \rightarrow G$ maps a rank $n-1$, free abelian, finite-index subgroup $G_{1}$ of $\widetilde{G}$ isomorphically onto a subgroup of $\widetilde{G_{1}} / F \leq G$. This proves Theorem 1.2(4), Lemma 2.19 and the entire Theorem [1.2, provided that $c_{2}(X)=0$ in $N^{2}(X)$.

Now Theorem 1.2 follows from the following:

Lemma 2.21. $c_{2}(X)=0$ in $N^{2}(X)$.

We prove the lemma. Suppose to the contrary that $c_{2}(X) \neq 0$ in $N^{2}(X)$. We shall show that this contradicts the minimality assumption on the pair $(X, G)$. There is an extremal birational contraction $\tau=\tau_{H}: X \rightarrow X_{H}$ corresponding to the rational polyhedral face

$$
F_{H}:=\left\{\alpha \in \overline{\mathrm{NE}}(X) \mid A_{H} \cdot \alpha=0\right\}
$$

of the closed cone of effective curves $\overline{\mathrm{NE}}(X)$ (cf. [2, Proof of Theorem 3.9.1]), so that $\tau(C)$ is a point for a curve $C$ on $X$ if and only if the class $[C] \in F_{H}$, and $A_{H}=\tau^{*} A_{H}^{\prime}$ (resp. $L_{g_{i}}=\tau^{*} L_{g_{i}}^{\prime}$ ) for an ample $\mathbb{R}$-divisor $A_{H}^{\prime}$ (resp. a nef $\mathbb{R}$ divisor $L_{g_{i}^{\prime}}$ ). By [22, Proof of Claim 2.11], $X_{H}$ has only canonical singularities and $K_{X_{H}} \sim 0$, because the same assertions are true for $X$. Since $A_{H}$ is the sum of nef $H$-eigenvectors, $F_{H}$ is $H$-stable. So the extremal contraction $\tau$ is $H$-equivariant.

Claim 2.22. $X_{H}$ has no $H$-periodic subvariety $D$ of dimension $s$ in $\{1, \ldots, n-1\}$. Hence every $H$-periodic subvariety of $X$ (especially the exceptional locus of $\tau_{H}$ ) is contracted to a point(s) by $\tau_{H}$.

Proof. We prove the claim. Since $\tau: X \rightarrow X_{H}$ is $H$-equivalent and $L_{g_{i}}=\tau^{*} L_{g_{i}}^{\prime}$, our $L_{g_{i}}^{\prime}$ and $L_{g_{i}}$ give rise to the same character $\chi_{i}$ on $H$. Now the proof of Lemma 2.18 shows that $\left(A_{H}^{\prime}\right)^{s} . D=0$. Since $A_{H}^{\prime}$ is ample, this contradicts Nakai's ampleness criterion (generalized to $\mathbb{R}$-divisors by Campana and Peternell). This proves Claim 2.22 .

We return back to the proof of Lemma 2.21

Take $u \in N(G)$ and set $H_{u}:=u^{-1} H u$. Then $G=N(G) H_{u}$ and it satisfies the four assertions of Theorem 2.2 (with $H$ replaced by $H_{u}$ ). Set $g_{i}^{\prime}:=u^{-1} g_{i} u$ so that $H_{u}=\left\langle g_{1}^{\prime}, \ldots, g_{n-1}^{\prime}\right\rangle$. Since $\left(g_{i}^{\prime}\right)^{*}\left(u^{*} L_{g_{i}}\right)=u^{*} g_{i}^{*}\left(u^{-1}\right)^{*}\left(u^{*} L_{g_{i}}\right)=d_{1}\left(g_{i}\right) u^{*} L_{g_{i}}$, we may take $L_{g_{i}^{\prime}}=u^{*} L_{g_{i}}$ and similarly $L_{g_{i}^{-1}}=u^{*} L_{g_{1}^{-1}}$ (cf. Lemma 2.13), which are all nef common $H_{u}$-eigenvectors. Set

$$
A_{H_{u}}=L_{g_{1}^{\prime}}+\cdots+L_{g_{n-1}^{\prime}}+L_{g_{1}^{\prime}-1}, \quad u^{*} F_{H}:=\left\{u^{*}(\alpha) \mid \alpha \in F_{H}\right\} .
$$


Then $A_{H_{u}}=u^{*} A_{H}$ and

$$
u^{*} F_{H}=F_{H_{u}}:=\left\{\alpha \in \overline{\mathrm{NE}}(X) \mid A_{H_{u}} \cdot \alpha=0\right\} .
$$

Since each $u^{*} F_{H}$ is spanned by finitely many extremal rays in the cone $\overline{\mathrm{NE}}(X)$, there are finitely many $u_{i} \in N(G)(i=1, \ldots, t)$ such that

$$
\begin{aligned}
F_{G}: & =\bigcap_{g \in G} g^{*} F_{H}=\bigcap_{u \in N(G)} u^{*} F_{H}=\bigcap_{i=1}^{t} u_{i}^{*} F_{H} \\
& =\left\{\alpha \in \overline{\mathrm{NE}}(X) \mid A_{H_{u_{i}}} \cdot \alpha=0,1 \leq i \leq t\right\}=\left\{\alpha \in \overline{\mathrm{NE}}(X) \mid \sum_{i=1}^{t} A_{H_{u_{i}}} \cdot \alpha=0\right\},
\end{aligned}
$$

where the second equality is true because $G=N(G) H$ and $A_{H}$ is the sum of nef $H$-eigenvectors.

Claim 2.23. $F_{G}=0$.

Proof. We prove the claim. Suppose to the contrary that $F_{G} \neq 0$. Note that $F_{G}$ is $G$-stable. As argued before Claim 2.22, there is a non-isomorphic $G$-equivariant birational extremal contraction $\tau_{G}: X \rightarrow X_{G}$ so that $\tau_{G}(C)$ is a point for a curve $C$ on $X$ if and only if the class $[C] \in F_{G}$. Also,

$$
A_{G}:=\sum_{i=1}^{t} A_{H_{u_{i}}}=\tau_{G}^{*} A_{G}^{\prime}, \quad L_{g_{i}^{\prime}}=\tau_{G}^{*} L_{g_{i}^{\prime}}^{\prime}
$$

for some ample divisor $A_{G}^{\prime}$ and nef divisor $L_{g_{i}^{\prime}}^{\prime}$ on $X_{G}\left(L_{g_{i}^{\prime}}\right.$, w.r.t. every given $u$, having been defined preceding this claim) and $X_{G}$ has at worst canonical singularities. The same proof of Claim 2.22 (applied to all $H_{u}$ ) and the ampleness of $A_{G}^{\prime}$ show that $X_{G}$ has no positive-dimensional $G$-periodic subvariety and hence $\operatorname{dim} \operatorname{Sing} X_{G} \leq 0$. This contradicts the minimality of the pair $(X, G)$. Therefore, $F_{G}=0$ and Claim 2.23 is true.

We return to the proof of Lemma 2.21, Let $\sigma: Z \rightarrow X$ be some Hironaka's blowup with centre in the exceptional locus of $\tau=\tau_{H}: X \rightarrow X_{H}=: Y$, such that $-E$ is $\tau \sigma$-relatively ample for some effective $\tau \sigma$-exceptional divisor $E$ on $Z$. Since $A=\tau^{*} A^{\prime}$ for some ample divisor $A^{\prime}$ on $Y$ (with $A:=A_{H}$ and $\left.A^{\prime}:=A_{H}^{\prime}\right), \sigma^{*} A-E$ is an ample divisor on $Z$ if $A$ is replaced by a positive multiple (cf. [10, Proposition 1.45]). By Claim 2.22 and the construction of the blowup $\sigma$, we have $\operatorname{dim} \tau \sigma(E) \leq 0$, so $\sigma^{*} A . E=(\tau \sigma)^{*} A^{\prime} . E=0$. The non-vanishing of $c_{2}(X)$ in $N^{2}(X)$ and Lemma 2.16 imply the existence of a non-zero effective 1-cycle $C:=c_{2}(X) \cdot M_{1} \ldots M_{n-3}$. Since ampleness is an open condition, replacing $M_{i}$ by a small positive multiple we may write

$$
\sigma^{*} A-E=\sigma^{*} M_{i}+P_{i}
$$

for some ample $\mathbb{R}$-divisor $P_{i}$. Since the difference $\sigma_{*} c_{2}(Z)-c_{2}(X)$ (as $(n-2)$ cycles) lies in the centre of the blowup of $\sigma: Z \rightarrow X$ (i.e., in the exceptional locus of $\tau: X \rightarrow Y$ by the construction of $\sigma$ ) and hence is contracted by $\tau$ to a finite subset of $Y$, and since $A=\tau^{*} A^{\prime}$, we have $\sigma_{*} c_{2}(Z) . A=c_{2}(X) \cdot A$ as $(n-3)$-cycles on $X$.

Consider the 1-cycle $\ell\left(i_{1}, \ldots, i_{s}\right):=\sigma_{*}\left(c_{2}(Z) . P_{i_{1}} \ldots P_{i_{s}}\right) A^{n-s-3}$ on $X$. Since $\sigma: Z \rightarrow X$ is isomorphic outside the exceptional locus of $\tau: X \rightarrow Y$ by the construction and the intersection of $c_{2}(X)$ with $\operatorname{Nef}(X) \times \cdots \times \operatorname{Nef}(X)(n-3$ of them) 
lies in $\overline{\mathrm{NE}}(X)$ (Miyaoka's pseudo-effectivity), our $\ell\left(i_{1}, \ldots, i_{s}\right)=\ell_{1}\left(i_{1}, \ldots, i_{s}\right)+$ $\ell_{2}\left(i_{1}, \ldots, i_{s}\right)$, with $\ell_{1}$ effective and $\ell_{2}$ supported on the exceptional locus of $\tau$, and hence $\ell_{2}\left(i_{1}, \ldots, i_{s}\right) \cdot A=\ell_{2}\left(i_{1}, \ldots, i_{s}\right) \cdot \tau^{*} A^{\prime}=0$ (cf. Claim 2.22).

Now Miyaoka's pseudo-effectivity of $c_{2}$ for minimal variety, the construction of the pseudo-effective sequence in Lemma 2.16, $\sigma_{*} c_{2}(Z) . A=c_{2}(X) . A$, the projection formula, $\sigma^{*} A . E=0$ and Lemma 2.18 imply:

$$
\begin{aligned}
0 & \leq c_{2}(X) \cdot M_{1} \ldots M_{n-3} \cdot A=\sigma_{*} c_{2}(Z) \cdot M_{1} \ldots M_{n-3} \cdot A \\
& =c_{2}(Z) \cdot \sigma^{*} M_{1} \ldots \sigma^{*} M_{n-3} \cdot \sigma^{*} A \\
& =c_{2}(Z) \cdot\left(\sigma^{*} A-P_{1}-E\right) \ldots\left(\sigma^{*} A-P_{n-3}-E\right) \cdot \sigma^{*} A \\
& =c_{2}(Z) \cdot\left(\sigma^{*} A-P_{1}\right) \ldots\left(\sigma^{*} A-P_{n-3}\right) \cdot \sigma^{*} A \\
& =c_{2}(Z) \cdot\left(\sigma^{*} A\right)^{n-2}-\sum_{i_{1}, \ldots, i_{s}}\left(c_{2}(Z) \cdot P_{i_{1}} \ldots P_{i_{s}}\right)\left(\sigma^{*} A\right)^{n-s-2} \\
& =\sigma_{*} c_{2}(Z) \cdot A^{n-2}-\sum_{i_{1}, \ldots, i_{s}} \sigma_{*}\left(c_{2}(Z) \cdot P_{i_{1}} \ldots P_{i_{s}}\right) A^{n-s-2} \\
& =c_{2}(X) \cdot A^{n-2}-\sum_{i_{1}, \ldots, i_{s}}\left(\ell_{1}\left(i_{1}, \ldots, i_{s}\right)+\ell_{2}\left(i_{1}, \ldots, i_{s}\right)\right) A \\
& =c_{2}(X) \cdot A^{n-2}-\sum_{i_{1}, \ldots, i_{s}} \ell_{1}\left(i_{1}, \ldots, i_{s}\right) \cdot A \leq c_{2}(X) \cdot A^{n-2}=0 .
\end{aligned}
$$

Thus the effective 1-cycle $C=c_{2}(X) \cdot M_{1} \ldots M_{n-3}$ satisfies $C \cdot A_{H}=0$ (with $A=$ $A_{H}$ ). Since $C$ is a $G$-eigenvector (cf. Lemma 2.16), we then have C.u $u_{i}^{*} A_{H}=0$ (with $u_{i}^{*} A_{H}=A_{H_{u_{i}}}$ ) for all $i$. Hence $C \in F_{G}=0$. This contradicts $C \neq 0$ (cf. Lemma 2.16). We have completed the proof of Lemma 2.21 and also Theorem 1.2 .

2.24. Proof of Theorem 2.1. We will use the argument prior to Section 2.14, We may and will freely replace $G$ by its finite-index subgroups. We may assume that $G=N(G) H$ with $H=\left\langle g_{1}, \ldots, g_{n-1}\right\rangle$ so that $H \mid H^{1,1}(X) \cong \mathbb{Z}^{\oplus n-1}$ as in Theorem 2.2 and satisfies the four assertions there. We use the notation in the proof of Theorem 2.2 and let

$$
A_{H}:=L_{g_{1}}+\cdots+L_{g_{n-1}}+L_{g_{1}^{-1}}
$$

be the nef and big class, where $L_{g_{j}^{ \pm}} \in \overline{K(X)}$ can be chosen to be common eigenvectors of $H$ since $H \mid H^{1,1}(X)$ is commutative.

As in Lemma 2.18, utilizing [13, Lemma 2.2] (writing the nef and big class $A_{H}$ as the sum of a Kähler class and a positive real current), $K_{X}$ equals zero (cohomologously). Thus there is an étale finite Galois covering $\widetilde{X}=T \times S \times Y \rightarrow X$ such that $T$ is a complex torus, $S$ is a product of hyperkähler manifolds $S_{i}, Y$ is a product of (projective) Calabi-Yau manifolds $Y_{j}$, and $G$, replaced by its finite-index subgroup, lifts to some group

$$
\widetilde{G} \leq \operatorname{Aut}(T) \times \prod \operatorname{Aut}\left(S_{i}\right) \times \prod \operatorname{Aut}\left(Y_{j}\right)
$$

with $\widetilde{G} / \operatorname{Gal}(\widetilde{X} / X)=G(\operatorname{cf}$. [1, $\S 3])$. As in Theorem [1.2, $N(\widetilde{G})$ is the preimage of $N(G)$, via the quotient map $\widetilde{G} \rightarrow G$, so that $\widetilde{G} / N(\widetilde{G})=\mathbb{Z}^{\oplus n-1}$, and hence the $\operatorname{rank} r(\widetilde{G})=n-1$. Since the projections of $\widetilde{X}$ to its factors $T, S_{i}$ and $Y_{j}$ are $\widetilde{G}$-equivariant, the maximality of $r(\widetilde{G})$ implies that $\widetilde{X}$ equals $T, S=S_{1}$ or $Y=Y_{1}$, 
i.e., is a complex torus, a hyperkähler manifold, or a projective Calabi-Yau manifold (cf. [19, Lemma 2.10]).

Since $X$ is non-algebraic, so is $\widetilde{X}$, and hence $\widetilde{X}$ equals $T$ or $S$. If $\widetilde{X}$ is hyperkähler, then we reach a contradiction: $2 \leq n-1=r(\widetilde{G}) \leq 1$ (cf. [9, Theorem 4.6]). Thus $\widetilde{X}$ is a complex torus. Now the argument of Lemma 2.19 (easier now) implies Theorem 2.1 .

\section{ACKNOWLEDGEMENT}

The author would like to thank Frederic Campana for pointing out the algebraicity of the Calabi-Yau factors in the Bogomolov decomposition.

\section{REFERENCES}

[1] Arnaud Beauville, Some remarks on Kähler manifolds with $c_{1}=0$, Classification of algebraic and analytic manifolds (Katata, 1982), Progr. Math., vol. 39, Birkhäuser Boston, Boston, MA, 1983, pp. 1-26. MR728605 (86c:32031)

[2] Caucher Birkar, Paolo Cascini, Christopher D. Hacon, and James McKernan, Existence of minimal models for varieties of log general type, J. Amer. Math. Soc. 23 (2010), no. 2, 405-468, DOI 10.1090/S0894-0347-09-00649-3. MR2601039 (2011f:14023)

[3] Garrett Birkhoff, Linear transformations with invariant cones, Amer. Math. Monthly 74 (1967), 274-276. MR0214605 (35 \#5454)

[4] F. Campana, F. Wang and D.-Q. Zhang, Automorphism groups of positive entropy on projective threefolds, Trans. Amer. Math. Soc. 366 (2014), no. 3, 1621-1638. MR3145744

[5] S. Cantat and A. Zeghib, Holomorphic actions of higher rank lattices in dimension three, Preprint 2009.

[6] Tien-Cuong Dinh and Nessim Sibony, Groupes commutatifs d'automorphismes d'une variété kählérienne compacte (French, with English and French summaries), Duke Math. J. 123 (2004), no. 2, 311-328, DOI 10.1215/S0012-7094-04-12323-1. MR2066940 (2005g:32020)

[7] Akira Fujiki, On automorphism groups of compact Kähler manifolds, Invent. Math. 44 (1978), no. 3, 225-258. MR.0481142 (58 \#1285)

[8] Yujiro Kawamata, Minimal models and the Kodaira dimension of algebraic fiber spaces, J. Reine Angew. Math. 363 (1985), 1-46, DOI 10.1515/crll.1985.363.1. MR814013 (87a:14013)

[9] JongHae Keum, Keiji Oguiso, and De-Qi Zhang, Conjecture of Tits type for complex varieties and theorem of Lie-Kolchin type for a cone, Math. Res. Lett. 16 (2009), no. 1, 133-148. MR2480567(2010e:14039)

[10] János Kollár and Shigefumi Mori, Birational geometry of algebraic varieties, Cambridge Tracts in Mathematics, vol. 134, Cambridge University Press, Cambridge, 1998. With the collaboration of C. H. Clemens and A. Corti. Translated from the 1998 Japanese original. MR.1658959(2000b:14018)

[11] David I. Lieberman, Compactness of the Chow scheme: applications to automorphisms and deformations of Kähler manifolds, Fonctions de plusieurs variables complexes, III (Sém. François Norguet, 1975-1977), Lecture Notes in Math., vol. 670, Springer, Berlin, 1978, pp. 140-186. MR521918 (80h:32056)

[12] Noboru Nakayama and De-Qi Zhang, Building blocks of étale endomorphisms of complex projective manifolds, Proc. Lond. Math. Soc. (3) 99 (2009), no. 3, 725-756, DOI 10.1112/plms/pdp015. MR2551469(2011c:14040)

[13] Noboru Nakayama and De-Qi Zhang, Polarized endomorphisms of complex normal varieties, Math. Ann. 346 (2010), no. 4, 991-1018, DOI 10.1007/s00208-009-0420-y. MR.2587100 (2011c:14103)

[14] Keiji Oguiso, Automorphisms of hyperkähler manifolds in the view of topological entropy, Algebraic geometry, Contemp. Math., vol. 422, Amer. Math. Soc., Providence, RI, 2007, pp. 173-185, DOI 10.1090/conm/422/08060. MR2296437(2008a:14055)

[15] Daniel Segal, Polycyclic groups, Cambridge Tracts in Mathematics, vol. 82, Cambridge University Press, Cambridge, 1983. MR713786 (85h:20003) 
[16] N. I. Shepherd-Barron and P. M. H. Wilson, Singular threefolds with numerically trivial first and second Chern classes, J. Algebraic Geom. 3 (1994), no. 2, 265-281. MR1257323 (95h:14033)

[17] Joseph A. Wolf, Growth of finitely generated solvable groups and curvature of Riemanniann manifolds, J. Differential Geometry 2 (1968), 421-446. MR0248688 (40 \#1939)

[18] De-Qi Zhang, Automorphism groups and anti-pluricanonical curves, Math. Res. Lett. 15 (2008), no. 1, 163-183. MR2367182 (2009f:14072)

[19] De-Qi Zhang, A theorem of Tits type for compact Kähler manifolds, Invent. Math. 176 (2009), no. 3, 449-459, DOI 10.1007/s00222-008-0166-2. MR2501294(2010d:32013)

[20] De-Qi Zhang, Dynamics of automorphisms on projective complex manifolds, J. Differential Geom. 82 (2009), no. 3, 691-722. MR2534992 (2010k:14078)

[21] De-Qi Zhang, The g-periodic subvarieties for an automorphism $g$ of positive entropy on a compact Kähler manifold, Adv. Math. 223 (2010), no. 2, 405-415, DOI 10.1016/j.aim.2009.08.010. MR2565534(2011a:32036)

[22] De-Qi Zhang, Automorphism groups of positive entropy on minimal projective varieties, Adv. Math. 225 (2010), no. 5, 2332-2340, DOI 10.1016/j.aim.2010.04.022. MR2680167 (2011m:14070)

[23] De-Qi Zhang, Algebraic varieties with automorphism groups of maximal rank, Math. Ann. 355 (2013), no. 1, 131-146, DOI 10.1007/s00208-012-0783-3. MR3004578

Department of Mathematics, National University of Singapore, 10 Lower Kent Ridge ROAD, Singapore 119076

E-mail address: matzdq@nus.edu.sg 